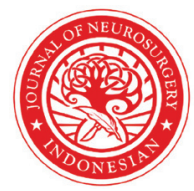

Indonesian Journal of Neurosurgery

\section{Decision making in cranioplasty: A case report}

\author{
Vayara Perumall Vinodh ${ }^{1 *}$, Sivakumaran Rajandran', \\ Theepan Sellamuthu Pulivendhan'², Pulivendhan Sellamuthu'
}

\title{
ABSTRACT
}

'Department of Neurosurgery, Sabah Brain \& Spine Centre, Hospital Queen Elizabeth 2, Sabah, Malaysia;

${ }^{2}$ Faculty of Medicine, Universiti

Kebangsaan Malaysia, Malaysia;

*Corresponding author:

Vayara Perumall Vinodh;

Department of Neurosurgery, Level 8 , Hospital Queen Elizabeth 2, Jalan Damai, 88300 Kota Kinabalu, Sabah, Malaysia; vinodh_uk@hotmail.com

Received: 2021-07-26

Accepted: 2021-10-28

Published: 2021-12-05
Introduction: Decompressive craniectomy (DC) is the treatment for intractable high intracranial pressure (ICP). Patients are left with a skull defect after the surgery which necessitates the need to close the defect.

Case presentation: We report a classic traumatic case that required DC and was followed by cranioplasty later. We explained in detail surgical steps which are considered paramount in avoiding complications. We also reviewed the relevant literature which aids surgeons in ideal decision making. Despite all the recent advances, there is no clear guideline on the ideal cranioplasty after DC. Cranioplasty is indicated to recreate the solid skull covering for protection from external injury. Cranioplasty has been proven beneficial in sunken flap syndromes that fail to respond to non-surgical treatment. Conclusion: Cranioplasty is a common procedure but involves many complications if not properly planned or done. Deliberate care must be taken during the perioperative period to prevent complications to ensure a good postoperative outcome.

Keywords: autologous bone flap, cranial defect, cranioplasty, decompressive craniectomy, surgical complication. Cite This Article: Vinodh, V.P., Rajandran, S., Pulivendhan, T.S., Sellamuthu, P. 2021. Decision making in cranioplasty: A case report. Indonesian Journal of Neurosurgery 4(3): 120-122. D0I: 10.15562/ijn.v4i3.202

\section{INTRODUCTION}

Decompressive craniectomy (DC) is still the treatment of choice for intractable high intracranial pressure (ICP) with mass effect secondary to traumatic brain injury (TBI) and stroke. ${ }^{1}$ Patients are left with a skull defect after the surgery and this necessitates the need to close back the defect after the acute period has passed to prevent secondary injuries due to exposed brain without a shield and also because it still remains as a cosmetic defect which needs correction.

Cranioplasty is the surgical intervention to repair cranial defects. There are various choices for cranioplasties which involve lifting the scalp and restoring the contour of the skull with either autografts or allografts.

\section{CASE PRESENTATION}

A 27-year-old car driver was involved in a traffic accident. He sustained severe TBI with a Glasgow Coma Scale (GCS) score of 8. An urgent computed tomography (CT) scan of the brain was performed which showed multiple contusions mainly at the bifrontal region with traumatic subarachnoid hemorrhage. There was a significant mass effect with obliterated basal cisterns and bifrontal DC was performed (Fig. 1). The bone flap was cryopreserved in a bone bank freezer.

He gradually improved after multidisciplinary team management and received active rehabilitation and physiotherapy. At discharge, his GCS score was 11 being E4V2M5. Five months after the first surgery, he was planned for autologous cranioplasty and was electively admitted for surgery. His operative site was not bulging and his scalp was free from infection with a well-healed surgical scar. He had an uneventful surgery and was discharged home three days later.

The bone flap is retrieved from the freezer and thawed at room temperature during surgery by placing it in sterile gentamycin solution before being washed with copious povidone mixed hydrogen peroxide solution and finally washed again in sterile gentamycin solution.

The patient was positioned supine and the head was turned so that the skull defect side is on top. The scalp was incised at the previous wound and dissected from the remaining cranium. The underlying dura

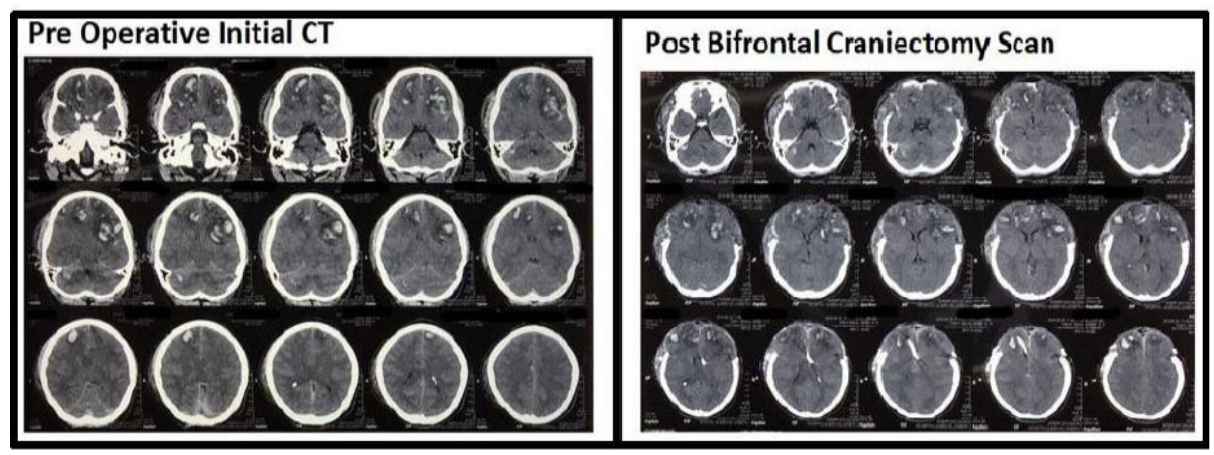

Figure 1. Pre-operative and post-operative CT scan images of the patient. 


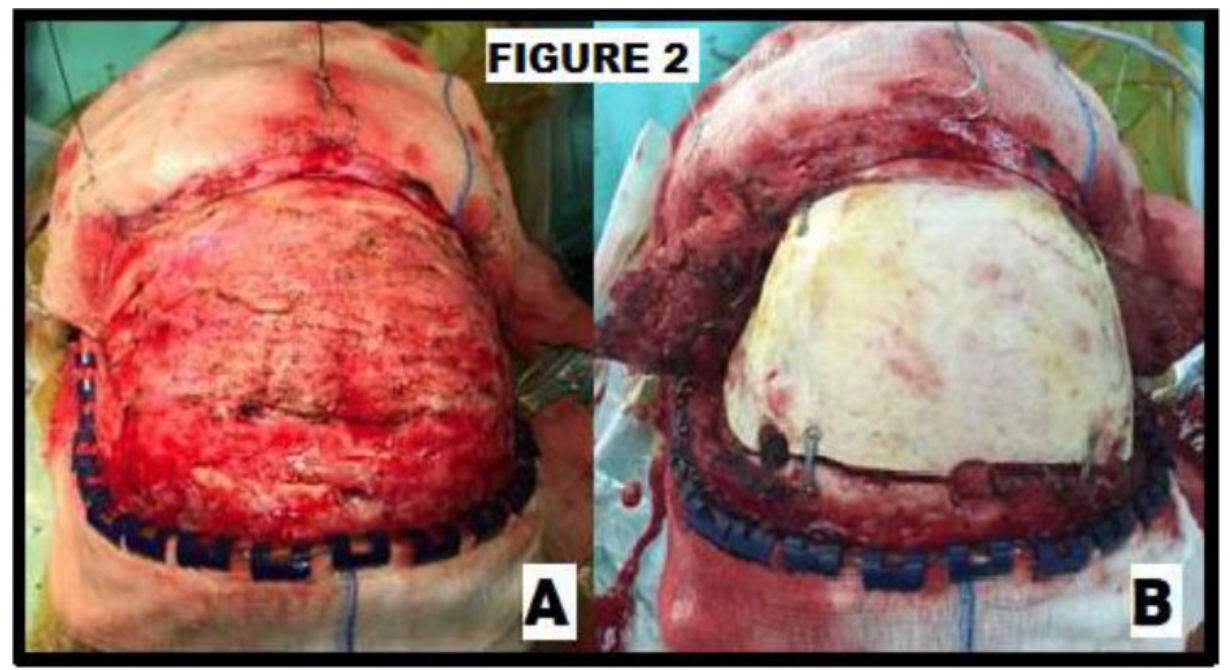

Figure 2. A. Careful dissection of the galea from the underlying dura; $B$. The placement of the bone flap with mini plates and screws.

Table 1. Criteria of the ideal cranioplasty.

\begin{tabular}{ll}
\hline \multicolumn{1}{c}{ Criteria } & \multicolumn{1}{c}{ Cranioplasty material features } \\
\hline Size of the defect & Must fit the cranial defect and achieve complete closure \\
Radiological property & Good radiolucency and MRI compatible \\
Biological safety & Resistance to infections or better if antibiotic coated \\
Biomechanical property & Resistance to heat and does not expand \\
Mould ability & Easy to shape but strong material \\
Affordability & Inexpensive \\
Origin & Inert but autologous origin is the best \\
\hline
\end{tabular}

was carefully dissected from the adherent galea to avoid any tear and cerebrospinal fluid (CSF) leak (Fig. 2A). Care must be taken to include the temporalis muscle in the raised flap either as a single flap or separately. Leaving behind temporalis muscle will cause suboptimal cosmetic benefit from cranioplasty with asymmetry and flattened temporalis fossa. Patients also often complain of pain while chewing in cases where temporalis muscle was not raised.

The edges of the skull at the previous craniectomy site were released of any adhesion using a periosteal elevator so that the cranium and the bone flap would fit. A final inspection of the dura was done to ensure the absence of defects with the CSF leak. The bone was then put into place and fixed to the skull using mini plates with screws for better cosmetic approximation (Fig. 2B). Suture ties or wire ties can also be used for this purpose. This was followed by careful closure of the surgical wound.

\section{DISCUSSION}

Craniotomy or skull trepanation along with corrective cranioplasty existed since ancient days with some dating back to 7000 BC. This procedure has seen tremendous evolution in the types of materials and techniques used. As the number of various ideas increases with different efforts being developed for the ease of the surgeon and the benefit of the patient. Despite all of these, there is no ideal implant if it is compared to the autologous bone flap because any other implant is considered a foreign material. An ideal cranioplasty must have the following features as seen in Table 1 but still, there is no perfect material to fit all these criteria. ${ }^{1,2}$

The decision of when to do the cranioplasty also matters as there is no clear guideline on the ideal timing of cranioplasty after craniectomy. A study of 157 patients, showed the least amount spent on research; new technologies are of complications were associated with a postoperative interval of fewer than 4 weeks or more than 20 weeks after DC, thus using both as acceptable timing for cranioplasty. This study concluded that if clinically safe and feasible, cranioplasty during the same hospitalization as DC for trauma can be undertaken without increasing complication risks. On the whole, the optimal timing of cranioplasty after DC still remains unknown. ${ }^{3}$

As most surgeons would avoid unnecessary surgeries especially in vegetative or bed-bound patients, cranioplasty is often indicated in DC patients with sunken flap syndrome causing neurologic deficits. A cranioplasty flap is required to prevent the atmospheric pressure to cause inward herniation of the skin flap leading to compression of the brain structures. This condition can be severe and cause irreversible damage if not identified and treated early. ${ }^{4}$

Cranioplasty is also indicated to recreate the solid skull covering that protects the brain from injury. It also cosmetically gives patients a near-normal skull appearance if possible. Post-DC patients are often required to wear helmets to protect them from further traumatic force especially during falls as the brain lacks a solid protective covering. Cranioplasty help protect the brain from external injury. ${ }^{5}$

Complications following cranioplasty can be divided into early and late. Early complications are hematoma, screw failure, implant breakage, and wound breakdown. Late complications are bone graft resorption, implant loosening, and implant extrusion. Infections and seizures can be both part of early and late complications. ${ }^{5}$ Surgeons should always ensure proper methods are undertaken in order to minimize or avoid these complications.

\section{CONCLUSION}

Cranioplasty is a common procedure but involves many complications if not properly planned or done. There is no absolutely ideal material for cranioplasty and the closest to the ideal is autologous bone flaps but the risk of bone absorption is always 
present. The ideal timing of cranioplasty is still debatable and is mostly based on the surgeon's preference. Deliberate care must be taken during the perioperative period to prevent complications to ensure a good postoperative outcome.

\section{CONFLICT OF INTEREST}

The authors declared no conflict of interest regarding the preparation of this manuscript.

\section{AUTHOR CONTRIBUTION}

The authors contributed equally to preparing this manuscript.

\section{FUNDING}

None.

\section{REFERENCES}

1. Aydin S, Kucukyuruk B, Abuzayed B, Aydin S, Sanus GZ. Cranioplasty: Review of materials and techniques. J Neurosci Rural Pract. 2011; 2(2): 162 - 7. https://doi.org/10.4103/0976$\underline{3147.83584}$.

2. Sanan A, Haines SJ. Repairing holes in the head: A history of cranioplasty. Neurosurgery. 1997; 40(3): 588 - 603. https://doi. org/10.1097/00006123-199703000-00033.

3. Piedra MP, Nemecek AN, Ragel BT. Timing of cranioplasty after decompressive craniectomy for trauma. Surg Neurol Int. 2014; 5: 25. https:// doi.org/10.4103/2152-7806.127762.
4. Khan NAJ, Ullah S, Alkilani W, Zeb H, Tahir H, Suri J. Sinking skin flap syndrome: Phenomenon of neurological deterioration after decompressive craniectomy. Case Rep Med. 208; 2018: 9805395. https://doi. org/10.1155/2018/9805395.

5. Fusetti S, Hammer B, Kellman R, Matula C, Strong EB, Di Ieva A. Cranial vault \& skull base - Special considerations: Cranioplasty (bone versus alloplast). AO Surgery Reference - AO Foundation. 2011. Available at: https:// surgeryreference.aofoundation.org/cmf/ trauma/skull-base-cranial-vault/furtherreading/cranioplasty-bone-versus-alloplast.

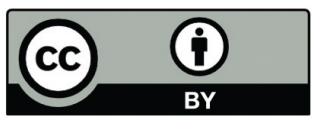

This work is licensed under a Creative Commons Attribution 